\title{
NREM sleep transient events in fronto-temporal dementia: beyond sleep stage archifecture
}

\author{
M. MAESTRI ${ }^{*}$, L. CARNICELLI ${ }^{*}$, N.-T. ECONOMOU², A. BONAKIS², \\ T. PAPARRIGOPOULOS², S.T. PAPAGEORGIOU², F.S. GIORGI', E. DI COSCIO', \\ G. TOGNONI ${ }^{1}$, R. FERRI ${ }^{3}$, U. BONUCCELLI', E. BONANNI ${ }^{1}$ \\ * These authors equally contributed to the paper \\ ${ }^{7}$ Neurology Unit, Department of Clinical and Experimental Medicine, University of Pisa, Via Roma \\ 67. Pisa, Italy; ${ }^{2}$ University of Athens Medical School, Sleep Study Unit, Eginition Hospital, Athens, \\ Greece; ${ }^{3}$ Department of Neurology IC, Oasi Institute for Research on Mental Retardation and \\ Brain Aging (IRCCS), Troina, Italy
}

\begin{abstract}
A B S T R A C T
Frontotemporal dementia (FTD) is increasingly becoming recognized as a major cause of early onset ( $<65$ years) neurodegenerative dementia. Although sleep disorders significantly impair patients' and caregivers' quality of life in neurodegenerative diseases, polysomnographic data in FTD patients are scarce in literature. Aim of our study was to investigate sleep microstructure in FTD, by means of Cyclic Alternating Pattern (CAP), in a group of ten behavioral variant FTD patients ( $6 \mathrm{M}, 4 \mathrm{~F}$; mean age $61.2 \pm 7.3$ years; disease duration: $1.4 \pm 0.7$ years) and to compare them with cognitively intact healthy elderly. Sleep in FTD patients was altered at different levels, involving not only the conventional sleep stage architecture parameters (total sleep time, single stage percentage, NREM/REM cycle organization), but also microstructure. FTD subjects showed CAP disruption with decreased slow wave activity related phases (A1 index, $n / h: 14.5 \pm 6.8$ vs $38.8 \pm 6.6 ; p<.001)$ and increased arousal-related fast CAP components (A2 index $22.9 \pm 8.2$

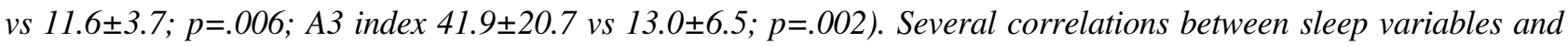
neuropsychological tests were found. Sleep impairment in FTD may be specifically related to the specific frontal lobe involvement in the neurodegenerative process. The pattern of alterations seems somewhat peculiar, probably due to the anatomical distribution of the neurodegenerative process with a major impact on frontal lobe generated sleep transients, and a substantial sparing of phenomena related to the posterior cortex.
\end{abstract}

Key words

Fronto-temporal dementia $\bullet$ Cyclic alternating pattern $\bullet$ NREM sleep instability $\bullet$ Neurodegeneration

\section{Introduction}

Frontotemporal dementia (FTD) is increasingly becoming recognized as a major cause of early onset ( $<65$ years) neurodegenerative dementia, being responsible for over $12 \%$ of cases (Harvey et al., 2003). Cognitive impairment in FTD mainly involves behavior, personality, and language, that combine into different clinical subtypes, that are behavioral and language variants. Approximately $60 \%$ of all FTD patients are affected by the behavioral variant of FTD (bvFTD) (Onyike and Diehl-Schmid, 2013), which is associated with more prominent social, personality and behavioral alterations, and can be identified with significant specificity through current clinical diagnostic criteria (Rascovsky et al., 2011). Among the cognitive and behavioral problems occurring in dementia, sleep disturbances often represent 
a major issue, significantly impairing the patient and the caregiver quality of life (Ancoli-Israel et al., 2006). Sleep has been evaluated extensively in Alzheimer 's Disease (AD), since it represents the most common form of dementia, and appears to be characterized mainly by nighttime fragmentation and increased latency; moreover, circadian disruption with daytime napping have been reported in both epidemiological and polysomnographic (PSG) studies (Vitiello et al., 1984; Bonanni et al., 2005; Guarnieri et al., 2012). Additionally, a more subtle involvement of short transient events that go beyond the classical sleep stage architecture has been reported also in preclinical phases, i.e. in amnestic mild cognitive impairment (Hita-Yañez et al., 2013; Maestri et al., 2015).

Conversely, sleep in FTD has been studied in much less depth. An Italian multicenter study reported a significant prevalence of sleep disorders in FTD, higher than in $\mathrm{AD}$, including insomnia, sleep disordered breathing, and excessive daytime sleepiness (Guarnieri et al., 2012). To the best of our knowledge, a few studies have assessed sleep specifically in FTD, by means of diaries, questionnaires, and actigraphy. Actigraphic pattern suggested highly fragmented circadian rhythms in both early stages and advanced FTD as compared to AD and controls while phase-delay observed by Anderson et al. (2009) was not replicated in a subsequent paper (Harper et al., 2001) dealing with a more severe population of institutionalized patients. A more recent observation (Merrilees et al., 2014) comparing 13 bvFTD with 9 patients with semantic dementia failed to highlight significant differences and showed normal sleep efficiency and timing of the nighttime sleep period, although clinical manifestations of bvFTD seem to impact more severely on caregiver sleep quality.

PSG data have been reported only in three papers (Pawlak et al., 1986; Kundermann et al., 2011; Bonakis et al., 2014), highlighting an early involvement of sleep architecture and an increased sleep fragmentation in FTD patients, compared to AD. However, this issue could be of particular interest, given the potential link between sleep, cognition, and frontal lobe functions, probably subserved by sleep-related synaptic plasticity (Ferini-Strambi et al., 2013; Mander et al., 2013, 2015; Feld et al., 2015). However, conventional sleep staging may not reveal subtle changes in sleep physiology, emphasizing the need for more sensitive parameters overcoming the standard 30-seconds epoch classification, and that have been encompassed under the concept of the so-called sleep microstructure. A comprehensive way to evaluate sleep microstructure is provided by the Cyclic Alternating Pattern (CAP) analysis, which has been also related to cognition, in both normal and cognitively impaired subjects (Parrino et al., 2012; Ferini-Strambi et al., 2013). CAP is a spontaneous and physiological endogenous rhythm of NREM sleep, characterized by EEG oscillations with a 20-40 second periodicity, underlying a condition of controlled and sustained arousal instability, that participates in the dynamic organization of sleep. CAP is composed of transient EEG arousal-related events (phases A of the cycle) separated by periods of background activity (phases B). Three main EEG patterns have been described for the A phases: predominantly synchronized slow waves in subtype A1, predominant EEG fast rhythms in A3, and a combination of both in A2. CAP sequences are present in normal NREM sleep, and the ratio of CAP time to NREM sleep time (CAP rate) has been proposed as a physiological marker of sleep instability, in both healthy and sleepdisordered individuals (Terzano et al., 2001; Parrino et al., 2006, 2012). The aims of the present study were to investigate CAP parameters in drug-naive FTD patients, to compare these parameters with those of cognitively intact elderly and to correlate these variables with neuropsychological testing scores within the bvFTD group.

\section{Methods}

\section{Subjects}

Subjects with behavioral-variant FTD (bvFTD) were recruited among out-patients in both Neurological Units in Athens and Pisa. Referral guidelines, clinical and laboratory workup, and diagnostic criteria have been described in detail elsewhere (Papageorgiou et al., 2009). Major organic disorders, other diseases that could influence normal sleep, subjective complaints of restless legs syndrome or symptomatic obstructive sleep apnea syndrome, were considered as exclusion criteria, as well as any previous psychiatric disturbances or use of drugs affecting the central nervous system. PSG-documented clinically relevant nocturnal breathing disorder (defined as Apnea Hypopnea Index greater than 15) was a further exclusion criterion. 


\section{MMSE and A3 in N2}

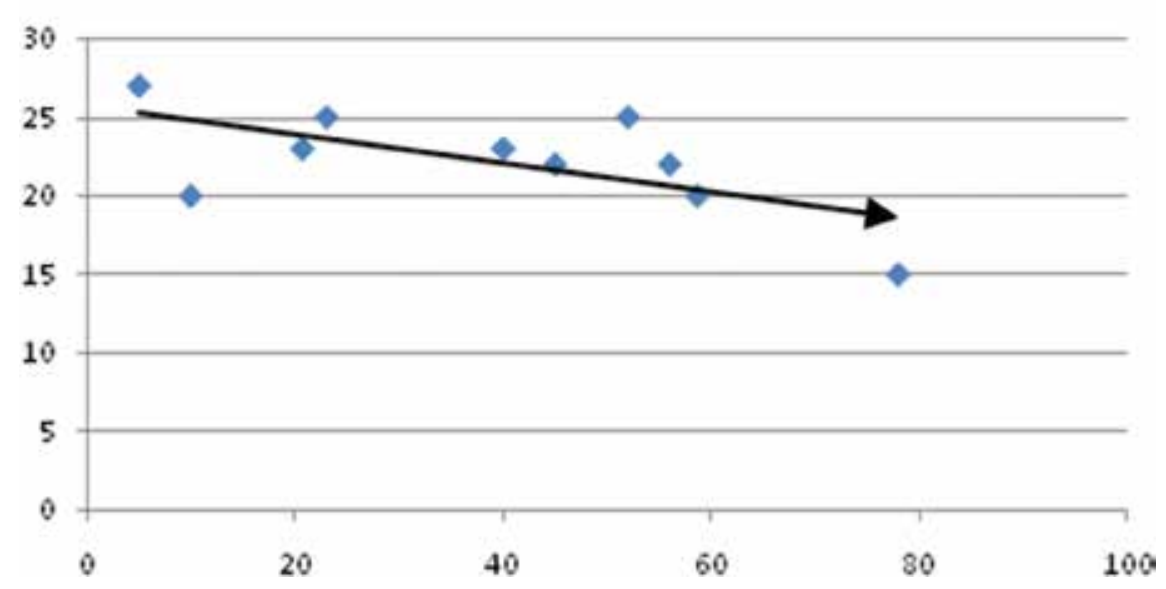

Fig. 1. - Correlation between Mini Mental State Examination-MMSE ( $y$-axis) and number of A3 in N2 (x-axis).

\section{MMSE and A3 Index}

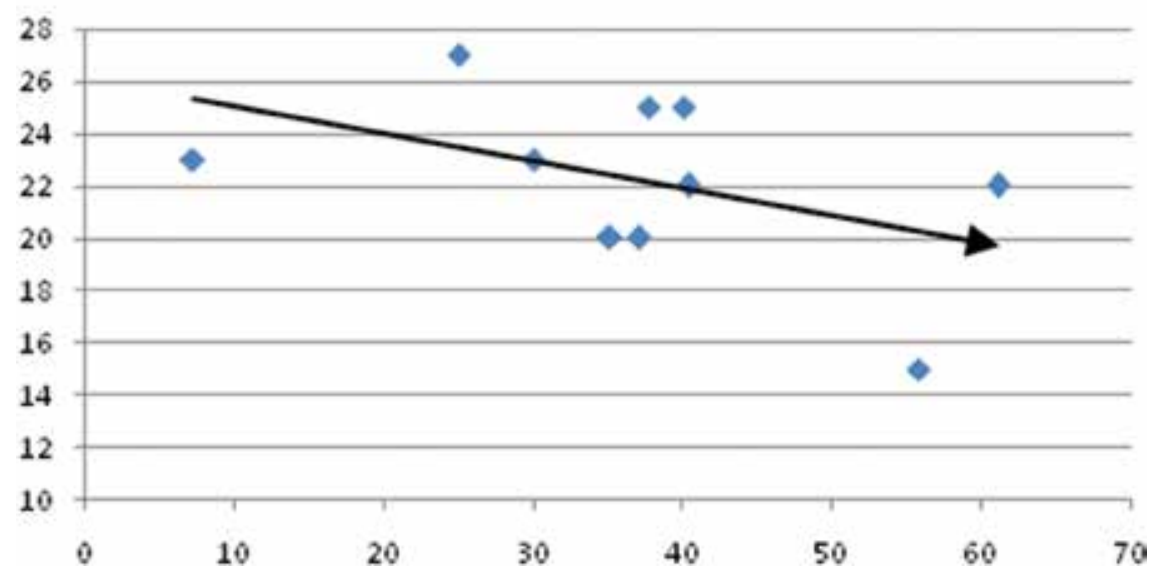

Fig. 2. - Correlation between Mini Mental State Examination-MMSE (y-axis) and A3 index (x-axis).

\section{MMSE and $A 3$ in SWS}

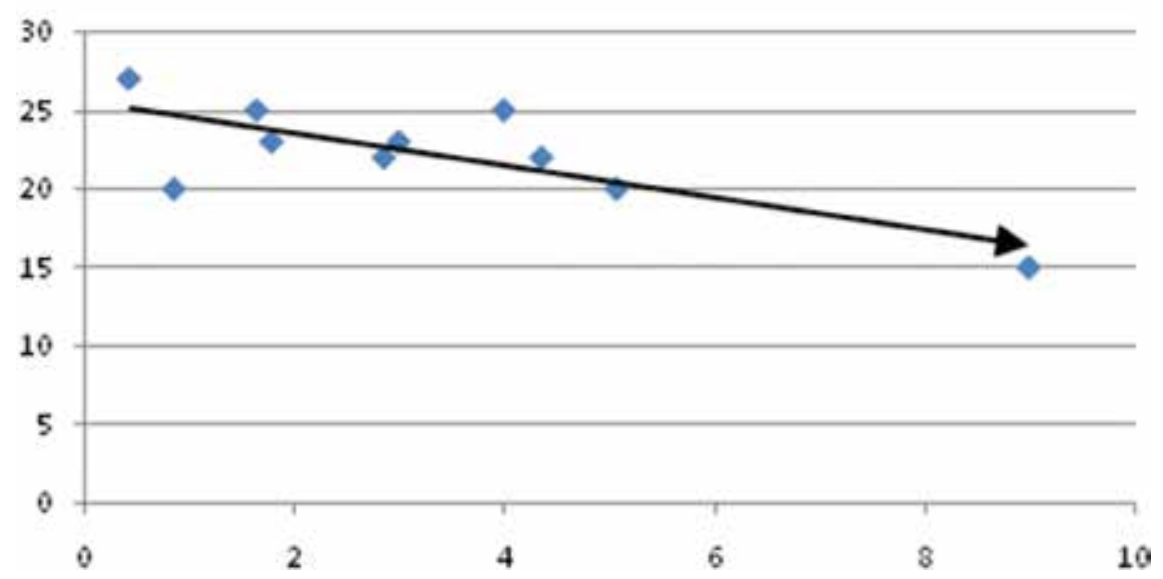

Fig. 3. - Correlation between Mini Mental State Examination-MMSE (y-axis) and number of A3 in slow wave sleep-SWS (x-axis). 
Ten patients with a diagnosis of behavioral-variant FTD (bvFTD) (6 men, 4 women; mean age 61.2 \pm 7.3 years; disease duration: $1.4 \pm 0.7$ years) were thus selected for the study. Twenty cognitively intact elderly (12 men, 8 women, mean age $70.2 \pm 12.5$ years), with the same inclusion and exclusion criteria, were used as a sex and age- ( $\mathrm{p}=$ N.S.) matched control group. Most of the patients and controls have been already enrolled in previous studies from our group (Bonakis et al., 2014; Maestri et al., 2015). Written informed consent was obtained from the participants and when needed by relatives, after detailed information on the study objectives and the research protocol was provided to them after scientific and ethics committee approval was obtained.

\section{Sleep evaluation}

Patients and controls underwent video-PSG examination including six-channel electro-encephalogram, (using two frontal, two central, and two occipital scalp electrodes referred to the contralateral mastoid (M1 or M2), two-channel electrooculogram, electromyogram of the submentalis and anterior tibialis muscles bilaterally, blood oxygen saturation through finger pulse oximeter, respiratory effort sensors placed over the rib cage and abdomen, snoring vibration sensor, nasal cannula pressure transducer, and thermistor.

Sleep architecture evaluation was based on the international scoring criteria by the American Academy of Sleep Medicine (Iber et al., 2007), with the elderly adjusted definition of slow wave sleep (Webb et al., 1982). Respiratory events and periodic leg movements were scored according to standard criteria: AHI (apnea-hypopnea index) and PLMS (periodic limb movements during sleep) index (Zucconi et al., 2006) were expressed as the number of events per hour of sleep (Zucconi et al., 2006; Iber et al., 2007). Sleep microstructure was evaluated according with the CAP analysis consensus guidelines (Terzano et al., 2001). CAP periods, with A phase subtypes (A1, A2, $\mathrm{A} 3$ ) and $\mathrm{B}$ phases, and non-CAP periods were identified. The following CAP parameters were obtained: CAP time (time spent in CAP), CAP rate (CAP time/ total NREM time*100), number and mean duration of $\mathrm{A} 1, \mathrm{~A} 2$ and $\mathrm{A} 3$ subtypes, number of CAP cycles, $\mathrm{nA} 1 / \mathrm{h}, \mathrm{nA} 2 / \mathrm{h}$ and $\mathrm{nA} 3 / \mathrm{h}$ indexes, mean duration of $\mathrm{B}$ phases, CAP sequence number and duration. Results of the visual analysis of CAP were computed by means of the program HypnoLab (SWS Soft, Italy).

\section{Neuropsychological assessment}

FTD subjects were administered a neuropsychological test battery that included:

- Mini Mental State Examination (MMSE, Folstein et al., 1975): MMSE is an 11 question tool routinely employed to assess mental status through five areas of cognitive function including orientation, registration, attention and calculation, recall, and language. Given a maximum score of 30 , a score of 23 or lower suggests cognitive impairment;

- Clock drawing test (Shulman et al., 1986): The clock-drawing test is a cognitive screening instrument that investigates a wide range of cognitive abilities including executive, visuoperceptual and constructional functions;

- Five words test (Dubois et al., 2002): It is a five-item immediate and delayed free- and, if necessary, cued-recall memory test. Scores are expressed as a total score (ranging from 0 to 10 ) by adding immediate free + immediate cued + delayed free + delayed cued recalls;

- Frontal assessment battery (FAB, Dubois et al., 2000): FAB is a brief and routinely employed tool that can be used at the bedside or in a clinic setting to assist in discriminating between dementias with a frontal dysexecutive phenotype and dementia of Alzheimer's Type;

- Frontal behavior inventory (FBI, Kertesz et al., 1997): FBI is a widely used caregiver focused tool that assesses FTD-Type Behavioral and Personality Changes through 24 items;

- Neuropsychiatric inventory (NPI, Cummings et al., 1994): NPI is an informed caregiver inventory that evaluates several domains of neuropsychiatric symptoms and psychopathology of patients with dementia and other neurodegenerative disorders;

- Semantic verbal fluency (Benton, 1968): This test, which is used in a wide range of neurodegenerative diseases, aims to assess the verbal ability and the executive functions.

\section{Statistical analysis}

The Mann-Whitney test was used to compare sleep architecture and microstructure parameters. The level of statistical significance adopted was 5\% $(\mathrm{p} \leq 0.05)$. The Spearman correlation coefficient was used to verify the dependency between neuropsy- 


\begin{tabular}{|l|c|c|c|}
\hline Table I. - Sleep structure parameters in FTD patients and controls. \\
\hline & FTD (\#10) & $\begin{array}{c}\text { HE } \\
(\# 20)\end{array}$ & $\begin{array}{c}\text { P } \\
\text { (Mann-Whitney-U test) }\end{array}$ \\
\hline Sleep onset, min & $36.0 \pm 26.1$ & $25.2 \pm 26.0$ & N.S. \\
\hline Total sleep time, min & $225.4 \pm 56.1$ & $401.5 \pm 68.0$ & $<0.001$ \\
\hline Sleep efficiency, $\%$ & $73.1 \pm 15.6$ & $82.1 \pm 13.8$ & N.S. \\
\hline N1, \% (TST) & $29.2 \pm 18.6$ & $7.8 \pm 3.2$ & 0.003 \\
\hline N2, \% (TST) & $50.5 \pm 14.6$ & $46.7 \pm 10.1$ & N.S. \\
\hline N3, \% (TST) & $9.1 \pm 5.3$ & $23.3 \pm 5.0$ & $<0.001$ \\
\hline REM, \% (TST) & $10.7 \pm 4.4$ & $22.2 \pm 7.1$ & $<0.001$ \\
\hline WASO, min & $87.6 \pm 46.5$ & $107.2 \pm 75.8$ & N.S. \\
\hline NREM/REM cycles, $n$ & $1.9 \pm 1.11$ & $3.8 \pm 1.3$ & N.S. \\
\hline Arousal/index, $n / h$ & $25.4 \pm 17.6$ & $20.2 \pm 8.5$ & \\
\hline $\begin{array}{l}\text { FTD=Frontotemporal dementia; HE=Healthy elderly; TST=Total sleep time; WASO=Wake after sleep onset. Data are presented } \\
\text { as mean value } \pm \text { standard deviation. }\end{array}$ &
\end{tabular}

chological performance and night sleep variables in FTD subjects, considering $\mathrm{p}<0.05$ as statistically significant.

\section{Results}

\section{General cognitive status}

FTD patients had a mean MMSE score of 21.8 \pm 3.6 and CDR was $1.3 \pm 0.6$.

\section{Sleep stage architecture}

FTD patients showed shorter total sleep time. Sleep structure was significantly impaired, as indicated by decreased sleep stages N3 and REM, compared with controls, while N1 was increased. Number of NREM/REM Cycles were decreased in FTD patients (table 1).

\section{CAP parameters}

Compared to cognitively intact controls, CAP slow components (A1) were decreased in FTD, both as percentage and absolute value (A1 index). FTD subjects had increased arousal-related fast CAP components (A2 and A3 subtypes), both as index and percentage.

Total CAP rate in whole NREM sleep was not different from controls, whereas CAP rate in N2 was significantly increased in FTD patients. A1 and A3 mean durations were decreased in FTD subjects, without significant change in cycle dura- tion. Moreover, CAP sequences showed increase in duration, and a reduction of their number. A trend of increased number of CAP cycles within sequences was also found (Table 2).

\section{Correlations}

As sleep architecture is concerned, the neuropsychiatric inventory score showed a positive correlation with wake after sleep onset $(\mathrm{min})$, and an inverse correlation with sleep efficiency and N3\%. Delayed Word Recall performance correlated with NREM/ REM cycles and, negatively, with N3\%.

MMSE score showed significant negative correlations with CAP total A3, A3 index, A3 in SWS and A3 in N3 (Figures 1-3). NPI score showed a negative correlation with nA1 in SWS, whereas nA3 in N2 positively correlated with FBI and FAB scores.

The Clock Drawing Test score significantly correlated with nA1 in N2, whereas Verbal Semantic Fluency was correlated with nA1 in SWS, and negatively with nA2 in SWS; Delayed Word Recall inversely correlated with nA2 and nA3 in N1 (Table 3).

\section{Discussion}

To the best of our knowledge, only few studies on PSG- recorded nocturnal sleep in FTD are reported in literature. Among these studies, Pawlak et al. (1986) pointed out a reduction of total sleep time and slow wave sleep, compared with healthy elderly controls. Kundermann et al. (2011) reported 


\begin{tabular}{|c|c|c|c|}
\hline & $\begin{array}{l}\text { FTD } \\
(\# 10)\end{array}$ & $\begin{array}{l}\mathrm{HE} \\
(\# 20)\end{array}$ & $\begin{array}{c}\mathrm{P} \\
\text { (Mann-Whitney-U test) }\end{array}$ \\
\hline CAP rate, $\%$ & $55.3 \pm 13.9$ & $47.1 \pm 5.6$ & N.S. \\
\hline CAP rate $\mathrm{N} 1, \%$ & $35.4 \pm 16.0$ & $29.2 \pm 15.4$ & N.S. \\
\hline CAP rate $\mathrm{N} 2, \%$ & $65.1 \pm 17.1$ & $42.2 \pm 6.7$ & 0.005 \\
\hline CAP rate $\mathrm{N} 3, \%$ & $54.9 \pm 26.3$ & $61.5 \pm 10.4$ & N.S. \\
\hline Tot Num A1, \% & $19.5 \pm 6.9$ & $60.2 \pm 9.3$ & $<0.001$ \\
\hline Tot Num A2, \% & $29.5 \pm 10.1$ & $18.4 \pm 4.3$ & 0.019 \\
\hline Tot Num A3, \% & $51.0 \pm 13.7$ & $21.4 \pm 10.4$ & 0,001 \\
\hline Tot Num (A2+A3), \% & $80.5 \pm 6.9$ & $39.8 \pm 9.3$ & $<0.001$ \\
\hline Al Index, n/h NREM & $14.5 \pm 6.8$ & $38.8 \pm 6.6$ & $<0.001$ \\
\hline A2 Index, n/h NREM & $22.9 \pm 8.2$ & $11.6 \pm 3.7$ & 0.006 \\
\hline A3 Index, n/h NREM & $41.9 \pm 20.7$ & $13.0 \pm 6.5$ & 0.002 \\
\hline A mean duration, sec & $8.2 \pm 1.0$ & $7.5 \pm 1.8$ & N.S. \\
\hline Al mean duration, sec & $5.9 \pm 1.0$ & $6.2 \pm 1.4$ & 0.01 \\
\hline A2 mean duration, sec & $9,2 \pm 1,1$ & $8.9 \pm 2.8$ & N.S. \\
\hline A3 mean duration, sec & $9.1 \pm 1.8$ & $10.9 \pm 5.0$ & 0.031 \\
\hline CAP cycle duration, sec & $29.0 \pm 4.8$ & $31.1 \pm 2.3$ & N.S. \\
\hline Cycles in Sequence,n & $11.9 \pm 5.2$ & $8.0 \pm 0.9$ & N.S. \\
\hline Sequence duration,sec & $296.7 \pm 102.4$ & $216.0 \pm 26.4$ & 0.05 \\
\hline Sequence number, $\mathrm{n}$ & $23.3 \pm 5.7$ & $40.4 \pm 8.3$ & $<0.001$ \\
\hline
\end{tabular}

no significant PSG differences between FTD and $\mathrm{AD}$, although a statistical trend toward a reduced REM sleep in AD was found. It should be noted, however, that these authors did not discriminate between different FTD subtypes. In a previous work by our group (Bonakis et al., 2014), we described a disturbed sleep pattern in bv-FTD subjects, even at a very early stage, when compared to Alzheimer disease. Compared to healthy elderly, both bv-FTD and AD show decreased total sleep time, NREM/REM cycles, sleep stage N2 and REM, and increased N1. The findings reported here confirm these earlier data in a selected bv-FTD population, free from clinically relevant sleep breathing disorder, and add further elements, such as the significant reduction in N3, that correlate with neuropsychological test.

Many recent lines of evidence suggest that the medial prefrontal cortex may represent the most critical structure for the generation of slow wave activity and support a change of paradigm from the classic global sleep regulation (Borbely et al., 1982) to a concept of a local sleep regulation (Nobili et al., 2012; Halász et al., 2014). An EEG-functional magnetic resonance imaging study
(Dang-Vu et al., 2008) reported significant increases in activity specific to the medial prefrontal, inferior frontal, precuneus, and posterior cingulate areas, temporally associated with slow waves $(>140 \mathrm{uV})$ and delta waves (70-140 uV) during slow wave sleep.

Moreover, sleep disruption is associated with a wide range of cognitive, behavioral and mood impairment, among which frontal lobe dysfunction may have a major role (Goel et al., 2009). In this model, a two way relationship between slow wave activity during sleep and prefrontal cortex has been proposed, based on data from both healthy subjects and patients, that suggests a role for slow wave sleep in restoring prefrontal cortex functioning. A recent paper (Mander et al., 2013) has reported that even in cognitively intact elderly, subtle structural brain changes occurring in the prefrontal cortex might contribute to slow-wave activity disruption, and correlate with the age-related cognitive decline. Prefrontal cortex degeneration, as well as a significant impairment of its functioning as assessed by functional neuroimaging, has been very often described in FTD patients, and especially in bvFTD 


\begin{tabular}{|c|c|c|c|}
\hline \multicolumn{4}{|c|}{$\begin{array}{l}\text { Table III. - Spearman correlation coefficients between quantitative sleep macrostructural and microstructural parameters } \\
\text { and neuropsychological scores in subjects with bv-FTD. Only values with a p-level } \leq 0.05 \text { are reported. }\end{array}$} \\
\hline Sleep architecture & & $r$ & $\mathrm{p}$ \\
\hline Sleep efficiency, \% & NPI & $-0,70$ & 0,05 \\
\hline $\mathrm{N} 3, \%$ & Delayed Word Recall & $-0,75$ & 0,02 \\
\hline $\mathrm{N} 3, \%$ & NPI & $-0,85$ & 0,015 \\
\hline WASO, min & NPI & 0,70 & 0,05 \\
\hline NREM/REM cycles & Delayed Word Recall & 0,78 & 0,05 \\
\hline \multicolumn{4}{|l|}{ Sleep microstructure } \\
\hline $\mathrm{A} 1$ in $\mathrm{N} 2, \mathrm{n}$ & Clock Drawing Test & 0,78 & 0,04 \\
\hline $\mathrm{Al}$ in SWS, $\mathrm{n}$ & Verbal semantic fluency & 0,79 & 0,04 \\
\hline $\mathrm{Al}$ in SWS, $\mathrm{n}$ & NPI & $-0,76$ & 0,05 \\
\hline $\mathrm{A} 2$ in $\mathrm{N} 1, \mathrm{n}$ & Delayed Word Recall & $-0,75$ & 0,05 \\
\hline A2 in SWS, $n$ & Verbal semantic fluency & $-0,84$ & 0,02 \\
\hline $\mathrm{A} 3$ in $\mathrm{N} 1, \mathrm{n}$ & Delayed Word Recall & $-0,76$ & 0,04 \\
\hline A3 in N2, n & $\mathrm{FBI}$ & 0,82 & 0,08 \\
\hline $\mathrm{A} 3$ in $N 2, n$ & FAB & 0.75 & 0.03 \\
\hline Total A3, n & MMSE & $-0,69$ & 0,04 \\
\hline A3 Index, n/h NREM & MMSE & $-0,84$ & 0,01 \\
\hline A3 in SWS, $n$ & MMSE & $-0,78$ & 0,01 \\
\hline $\mathrm{A} 3$ in $\mathrm{N} 2, \mathrm{n}$ & MMSE & $-0,75$ & 0,02 \\
\hline
\end{tabular}

(Piguet et al., 2011): this might at least in part justify the above mentioned sleep alterations.

In our population, we found a correlation between NREM/REM cycle organization and verbal memory test. These results are in line with previous reports in healthy subjects both in young adults during experimental condition and in elderly subjects (Mazzoni et al., 1999; Ficca et al., 2000; Conte et al., 2012). Our data suggest that sleep in FTD patients is altered at different levels, involving not only the conventional sleep stage architecture parameters (total sleep time, single stage percentage, NREM/REM cycle organization), but also microstructure, as shown by our CAP analysis. Even if the global measure of sleep microstructure (CAP rate) was not different between FTD and controls, CAP was organized in fewer but longer sequences in FTD, suggesting a peculiar pattern of more sustained sleep instability. In other words, it seems that the CAP process begins less frequently in FTD patients, but once started it is less prone to terminate. The increased duration of CAP sequences might be related to the aging process per se (Smerieri et al., 2007; Parrino et al., 2012) but the reduction of number is similar to the results obtained in patients with Alzheimer disease (Maestri et al., 2015). Regarding CAP subtypes, A1 (i.e. CAP slow components) were reduced in FTD. This is in line with evidence suggesting that A1 are the expression of transient frontal cortex activation, whereas A2 and A3 mainly map to the parieto-occipital cortex (Ferri et al., 2005). A1 show a progressive decrease from adolescence to young adulthood, a plateau between young adults and middle aged, and then another drop from adulthood to senescence (Parrino et al., 1998; Parrino et al., 2012). In the physiological architecture of sleep, the A1 subtypes prevail in the build-up and maintenance of deep NREM sleep (Terzano et al., 2001). Decreased A1 subtypes may represent one of the pathophysiological mechanisms through which neurodegeneration leads to the disruption of NREM sleep, and specifically N3, build-up. The reduction of A1 may indicate a loss of protection of sleep continuity. CAP plays an important role in modulating the response to external and internal stimuli that may disturb sleep, with a balance between sleep protection and arousability. CAP A1 (slow high-amplitude EEG patterns) indicates sleep continuity while A2 and A3 
(fast low-voltage EEG) are indexes of sleep disruption, in agreement with their different behavioral and autonomic counterparts. Thus, the reduction in A1 subtypes might unbalance this mechanism with a resulting shift towards higher levels of arousability and lesser ability to maintain sleep continuity, in response to environmental and internal stimuli (Parrino et al., 2006; Halász et al., 2014).

In this study, the correlations between CAP parameters and the neuropsychological scores (mainly related to behavioral and executive functions) are in line with previous reports regarding a positive correlation between A1 subtypes and better cognitive functioning, as opposed to a worse performance predicted by A2 and A 3 subtypes. The first studies reporting a positive correlation between $\mathrm{A} 1$ and cognitive parameters involved a young individual with superior memory performance and an increased CAP A1\% (Ferini-Strambi et al., 2004) and participants to an experimental motor learning task who showed an increase in the number of A1 which was correlated with improvement in task performance (Ferri et al., 2008).

Since then, additional evidence relating CAP to learning processes and cognitive function has come mainly from experimental models and from pediatric populations with mental disability (Down syndrome, Fragile-X syndrome, attention-deficit/hyperactivity disorder (ADHD), pervasive developmental disorders) (Parrino et al., 2012; Ferini-Strambi et al., 2013; Novelli et al., 2013). It is worth to note that also in young healthy subjects (Aricò et al., 2010) a significant positive correlation has been reported between A1 subtypes and neuropsychological tests targeting frontal lobe cognitive functions (e.g., verbal fluency, working memory, verbal learning).

The impact of sleep alteration on behavioral aspects of FTD, as indicated by the correlation between sleep parameters and the neuropsychiatric inventory (NPI), may remind the clinical observation pointed out in schizophrenia, that, although traditionally considered as a distinct disorder, has been suggested to share a great deal of clinical, neuroimaging, pathological features with FTD (Momeni et al., 2010). Briefly, an involvement of the prefrontal cortex and a potential accelerated age-related decline in ventromedial prefrontal cortices in schizophrenia patients have been demonstrated (Unschuld et al., 2014; Zhang et al., 2014). Increased sleep fragmentation, sleep latency, wake after sleep onset (Chouinard et al., 2004) and specific NREM sleep abnormalities with decreased slow wave sleep have been reported and negatively correlated with symptoms severity and impairment of neurocognitive measures (Göder et al., 2006; Yang and Winkelman, 2006; Sarkar et al., 2010). As concerns CAP in schizophrenia, the only study performed (Ozone et al., 2013) highlighted in a small group of 7 patients treated with antipsychotics and benzodiazepines a marked reduction of A1\% while CAP rate is similar to reference data in young adults (Parrino et al., 2012).

In conclusion, in our group of patients with FTD, sleep is impaired also at the NREM microstructural level. The pattern of alterations seems somewhat peculiar, probably due to the anatomical distribution of the neurodegenerative process with a major impact on frontal lobe generated sleep transients, and a substantial sparing of phenomena related to the posterior cortex. Similarly to AD, also in FTD it is not clear if these alterations are just an epi-phenomenon or may also be a pathogenic mechanism of the neurodegenerative process (Landry et al., 2014). However, sleep disorders in FTD should always be taken into account because of their impact on quality of life of both patients and caregivers, and also because of the possible beneficial effect of treatment.

\section{References}

Ancoli-Israel S., Vitiello M.V. Sleep in dementia. Am. J. Geriatr. Psychiatry, 14: 91-94, 2006.

Anderson K.N., Hatfield C., Kipps C., Hastings M., Hodges J.R. Disrupted sleep and circadian patterns of frontotemporal dementia. Eur. J. Neurol., 16: 317-323, 2009.

Aricò D., Drago V., Foster P.S., Heilman K.M., Williamson J., Ferri R. Effects of NREM sleep instability on cognitive processing. Sleep Med., 11: 791-798, 2010.

Benton A.L. Differential behavioral effects in frontal lobe disease. Neuropsychologia, 6: 53-60, 1968.

Bonakis A., Economou N.T., Paparrigopoulos T., Bonanni E., Maestri M., Carnicelli L., Di Coscio E., Ktonas P., Vagiakis E., Theodoropoulos P., Papageorgiou C. Sleep in frontotemporal dementia is equally or possibly more disrupted, and at an earlier stage, when compared to sleep in Alzheimer's disease. Alzheimers Dis., 38: 85-91, 2014. 
Bonanni E., Maestri M., Tognoni G., Fabbrini M., Nucciarone B., Manca M.L., Gori S., Iudice A., Murri L. Daytime sleepiness in mild and moderate Alzheimer's disease and its relationship with cognitive impairment. J. Sleep Res., 14: 311-317, 2005.

Borbély A.A. A two process model of sleep regulation. Hum. Neurobiol., 1: 195-204, 1982.

Chouinard S., Poulin J., Stip E., Godbout R. Sleep in untreated patients with schizophrenia: a metaanalysis. Schizophr. Bull., 30: 957-967, 2004.

Cummings J.L., Mega M., Gray K., RosenbergThompson S., Carusi D.A., Gornbein J. The Neuropsychiatric Inventory: comprehensive assessment of psychopathology in dementia. Neurology, 44: 2308-2314, 1994.

Dang-Vu T.T., Schabus M., Desseilles M., Albouy G., Boly M., Darsaud A., Gais S., Rauchs G., Sterpenich V., Vandewalle G., Carrier J., Moonen G., Balteau E., Degueldre C., Luxen A., Phillips C., Maquet P. Spontaneous neural activity during human slow wave sleep. Proc. Natl. Acad. Sci. U.S.A., 30: 15160-15165, 2008.

Dubois B., Slachevsky A., Litvan I., Pillon B. The FAB: a Frontal Assessment Battery at bedside. Neurology, 55: 1621-1626, 2000.

Dubois B., Touchon J., Portet F., Ousset P.J., Vellas B., Michel B. "The 5 words": a simple and sensitive test for the diagnosis of Alzheimer's disease. Presse Med., 31: 1696-1699, 2002.

Feld G.B., Diekelmann S. Sleep smart-optimizing sleep for declarative learning and memory. Front. Psychol., 6: 622, 2015.

Ferini-Strambi L., Galbiati A., Marelli S. Sleep microstructure and memory function. Front. Neurol., 4: 159, 2013.

Ferini-Strambi L., Ortelli P., Castronovo V., Cappa S. Increased periodic arousal fluctuations during non-REM sleep are associated to superior memory. Brain Res. Bull., 63: 439-442, 2004.

Ferri R., Bruni O., Miano S., Terzano M.G. Topographic mapping of the spectral components of the cyclic alternating pattern (CAP). Sleep Med., 6: 29-36, 2005.

Ferri R., Huber R., Aricò D., Drago V., Rundo F., Ghilardi M.F., Massimini M., Tononi G. The slow-wave components of the cyclic alternating pattern (CAP) have a role in sleep-related learning processes. Neurosci. Lett., 432: 228-231, 2008.

Ficca G., Lombardo P., Rossi L., Salzarulo P. Morning recall of verbal material depends on prior sleep organization. Behav. Brain Res., 112: 159-163, 2000.
Folstein M.F., Folstein S.E., McHugh P.R. "Minimental state". A practical method for grading the cognitive state of patients for the clinician. $J$. Psychiatr. Res., 12: 189-198, 1975.

Göder R., Aldenhoff J.B., Boigs M., Braun S., Koch J., Fritzer G. Delta power in sleep in relation to neuropsychological performance in healthy subjects and schizophrenia patients. J. Neuropsychiatry Clin. Neurosci., 18: 529-535, 2006.

Goel N., Rao H., Durmer J.S., Dinges D.F. Neurocognitive consequences of sleep deprivation. Semin. Neurol., 29: 320-339, 2009.

Guarnieri B., Adorni F., Musicco M., Appollonio I., Bonanni E., Caffarra P., Caltagirone C., Cerroni G., Concari L., Cosentino F.I., Ferrara S., Fermi S., Ferri R., Gelosa G., Lombardi G., Mazzei D., Mearelli S., Morrone E., Murri L., Nobili F.M., Passero S., Perri R., Rocchi R., Sucapane P., Tognoni G., Zabberoni S., Sorbi S. Prevalence of sleep disturbances in mild cognitive impairment and dementing disorders: a multicenter Italian clinical cross-sectional study on 431 patients. Dement Geriatr. Cogn. Disord., 33:50-58, 2012.

Halász P., Bódizs R., Parrino L., Terzano M. Two features of sleep slow waves: homeostatic and reactive aspects--from long term to instant sleep homeostasis. Sleep Med., 15: 1184-1195, 2014.

Harper D.G., Stopa E.G., McKee A.C., Satlin A., Harlan P.C., Goldstein R., Volicer L. Differential circadian rhythm disturbances in men with Alzheimer disease and frontotemporal dementia. Arch. Gen. Psychiatry, 58: 353-360, 2001.

Harvey R.J., Skelton-Robinson M., Rossor M.N. The prevalence and causes of dementia in people under the age of 65 years. J. Neurol. Neurosurg. Psychiatry, 74:1206-1209, 2003.

Hita-Yañez E., Atienza M., Cantero J.L. Polysomnographic and Subjective Sleep Markers of Mild Cognitive Impairment. Sleep, 36: 1327-1334, 2013.

Iber C., Ancoli-Israel S., Chesson A.L., Jr., Quan S.F. for the American Academy of Sleep Medicine. The AASM manual for the scoring of sleep and associated events: rules, terminology and technical specifications. 1st ed 2007, Westchester, IL: American Academy of Sleep Medicine.

Kertesz A., Davidson W., Fox H. Frontal behavioral inventory: diagnostic criteria for frontal lobe dementia. Can. J. Neurol. Sci., 24: 29-36, 1997.

Kundermann B., Thum A., Rocamora R., Haag A., Krieg J.C., Hemmeter U. Comparison of polysomnographic variables and their relationship to cognitive impairment in patients with Alzheimer's 
disease and frontotemporal dementia. J. Psychiatr. Res., 45: 1585-1592, 2011.

Landry G.J., Liu-Ambrose T. Buying time: a rationale for examining the use of circadian rhythm and sleep interventions to delay progression of mild cognitive impairment to Alzheimer's disease. Front. Aging Neurosci., 6: 325, 2014.

Maestri M., Carnicelli L., Tognoni G., Di Coscio E., Giorgi F.S., Volpi L., Economou N.T., Ktonas P., Ferri R., Bonuccelli U., Bonanni E. Non-rapid eye movement sleep instability in mild cognitive impairment: A pilot study. Sleep Med., 16: 11391145, 2015.

Mander B.A., Marks S.M., Vogel J.W., Rao V., Lu B., Saletin J.M., Ancoli-Israel S., Jagust W.J., Walker M.P. $\beta$-amyloid disrupts human NREM slow waves and related hippocampus-dependent memory consolidation. Nat. Neurosci., 18: 1051-1057, 2015.

Mander B.A., Rao V., Lu B., Saletin J.M., Lindquist J.R., Ancoli-Israel S., Jagust W., Walker M.P. Prefrontal atrophy, disrupted NREM slow waves and impaired hippocampal-dependent memory in aging. Nat. Neurosci., 16: 357-364, 2013.

Mazzoni G., Gori S., Formicola G., Gneri C., Massetani R., Murri L., Salzarulo P. Word recall correlates with sleep cycles in elderly subjects. $J$. Sleep Res., 8: 185-188, 1999.

Merrilees J., Hubbard E., Mastick J., Miller B.L., Dowling G.A. Sleep in persons with frontotemporal dementia and their family caregivers. Nurs. Res., 63: 129-136, 2014.

Momeni P., Wickremaratchi M.M., Bell J., Arnold R., Beer R., Hardy J., Revesz T., Neal J.W., Morris H.R. Familial early onset frontotemporal dementia caused by a novel S356T MAPT mutation, initially diagnosed as schizophrenia. Clin. Neurol. Neurosurg., 112: 917-920, 2010.

Nobili L., De Gennaro L., Proserpio P., Moroni F., Sarasso S., Pigorini A., De Carli F., Ferrara M. Local aspects of sleep: observations from intracerebral recordings in humans. Prog. Brain Res., 199: 219-232, 2012.

Novelli L., Ferri R., Bruni O. Sleep cyclic alternating pattern and cognition in children: a review. Int. J. Psychophysiol., 89: 246-251, 2013.

Onyike C.U., Diehl-Schmid J. The epidemiology of frontotemporal dementia. Int. Rev. Psychiatry, 25: 130-137, 2013.

Ozone M., Kuroda A., Aoki K., Manabe T., Itoh H. Sleep structure in medicated schizophrenia: A pilot study using cyclic alternating pattern method. Sleep and Biological Rhythms, 11: 278-281, 2013.
Papageorgiou S.G., Kontaxis T., Bonakis A., Kalfakis N., Vassilopoulos D. Frequency and causes of earlyonset dementia in a tertiary referral center in Athens. Alzheimer Dis. Assoc. Disord., 23: 347-351, 2009.

Parrino L., Boselli M., Spaggiari M.C., Smerieri A., Terzano M.G. Cyclic alternating pattern (CAP) in normal sleep: polysomnographic parameters in different age groups. Electroencephalogr. Clin. Neurophysiol., 107: 439-450, 1998.

Parrino L., Ferri R., Bruni O., Terzano M.G. Cyclic alternating pattern (CAP): The marker of sleep instability. Sleep Med. Rev., 16: 27-45, 2012.

Parrino L., Halasz P., Tassinari C.A., Terzano M.G. CAP, epilepsy and motor events during sleep: The unifying role of arousal. Sleep Med. Rev., 10: 267285, 2006.

Pawlak C., Blois R., Gaillard J.M., Richard J. Sleep in Pick disease. Encephale, 12: 327-334, 1986.

Piguet O., Hornberger M., Mioshi O., Hodges J.R. Behavioural-variant frontotemporal dementia: diagnosis, clinical staging and management. Lancet Neurol., 10: 162-172, 2011.

Rascovsky K., Hodges J.R., Knopman D., Mendez M.F., Kramer J.H., Neuhaus J., van Swieten J.C., Seelaar H., Dopper E.G., Onyike C.U., Hillis A.E., Josephs K.A., Boeve B.F., Kertesz A., Seeley W.W., Rankin K.P., Johnson J.K., Gorno-Tempini M.L., Rosen H., Prioleau-Latham C.E., Lee A., Kipps C.M., Lillo P., Piguet O., Rohrer J.D., Rossor M.N., Warren J.D., Fox N.C., Galasko D., Salmon D.P., Black S.E., Mesulam M., Weintraub S., Dickerson B.C., Diehl-Schmid J., Pasquier F., Deramecourt V., Lebert F., Pijnenburg Y., Chow T.W., Manes F., Grafman J., Cappa S.F., Freedman M., Grossman M., Miller B.L. Sensitivity of revised diagnostic criteria for the behavioural variant of frontotemporal dementia. Brain, 134: 2456-2477, 2011.

Sarkar S., Katshu M.Z., Nizamie S.H., Praharaj S.K. Slow wave sleep deficits as a trait marker in patients with schizophrenia. Schizophr. Res., 124: 127-133, 2010.

Shulman K.I., Shedletsky R., Silver I. The challenge of time: clock drawing and cognitive function in the elderly. Int. J. Geriatr. Psychiatry, 1: 135-140, 1986.

Smerieri A., Parrino L., Agosti M., Ferri R., Terzano M.G. Cyclic alternating pattern sequences and non-cyclic alternating pattern periods in human sleep. Clin. Neurophysiol., 118: 2305-2313, 2007.

Terzano M.G., Parrino L., Smerieri A., Chervin R., Chokroverty S., Guilleminault C. et al. Atlas, rules, and recording techniques for the scoring of cyclic alternating pattern (CAP) in human sleep. Sleep Med., 2: 537-553, 2001. 
Unschuld P.G., Buchholz A.S., Varvaris M., van Zijl P.C., Ross C.A., Pekar J.J., Hock C., Sweeney J.A., Tamminga C.A., Keshavan M.S., Pearlson G.D., Thaker G.K., Schretlen D.J. Prefrontal brain network connectivity indicates degree of both schizophrenia risk and cognitive dysfunction. Schizophr. Bull., 40: 653-664, 2014.

Vitiello M.V., Bokan J.A., Kukull W.A., Muniz R.L., Smallwood R.G., Prinz P.N. Rapid eye movement sleep measures of Alzheimer's-type dementia patients and optimally healthy aged individuals. Biol. Psychiatry, 19: 721-734, 1984.

Webb W.B., Dreblow L.M. A modified method for scoring slow wave sleep of older subjects. Sleep, 5: 195-199, 1982.

Yang C., Winkelman J.W. Clinical significance of sleep EEG abnormalities in chronic schizophrenia. Schizophr. Res., 82: 251-260, 2006.
Zhang F., Qiu L., Yuan L., Ma H., Ye R., Yu F., Hu P., Dong Y., Wang K. Evidence for progressive brain abnormalities in early schizophrenia: a crosssectional structural and functional connectivity study. Schizophr. Res., 159: 31-35, 2014.

Zucconi M., Ferri R., Allen R., Baier P.C., Bruni O., Chokroverty S., Ferini-Strambi L., Fulda S., Garcia-Borreguero D., Hening W.A., Hirshkowitz M., Högl B., Hornyak M., King M., Montagna P., Parrino L., Plazzi G., Terzano M.G.; International Restless Legs Syndrome Study Group (IRLSSG). The official World Association of Sleep Medicine (WASM) standards for recording and scoring periodic leg movements in sleep (PLMS) and wakefulness (PLMW) developed in collaboration with a task force from the International Restless Legs Syndrome Study Group (IRLSSG). Sleep Med., 7: 175-183, 2006. 